\title{
A Study on the Interaction Between Morphological and Semantic Features of $X$-ful Derivatives from the Perspective of Construction Morphology
}

\author{
Xiaojing Chen*, Changyong Chen
}

Hangzhou Dianzi University, Hangzhou, China

*Corresponding author. Email: 444843826@qq.com

\begin{abstract}
Suffixes are important components in English word formation. "-FUL" as one of common suffixes, it can produce various derivatives. Based on the American Contemporary English Corpus(COCA), this paper studies the morphological and semantic features of $414 \mathrm{x}$-ful derivatives. In addition, it adopts the theory of construction morphology to explore the interaction between its morphological and semantic features. The research has three findings. Firstly, the bases of $\mathrm{x}$-ful derivatives may be nouns, adjectives and verbs, from which $\mathrm{x}$-ful nouns and $\mathrm{x}$-ful adjectives can be derived. Secondly, it is found that there are sixteen semantic meanings for $\mathrm{x}$-ful adjectives while only one for $\mathrm{x}$-ful nouns. Thirdly, the bases of $\mathrm{x}$-ful adjectives have four semantic categories while those of $\mathrm{x}$-ful nouns have eight. This research helps English learners understand the semantic schema of $x$-ful derivatives and correctly grasp internal structural meaning in order to master such vocabulary.
\end{abstract}

Keywords: $x$-ful derivatives, Morphological features, Semantic features, Construction morphology, Interaction between morphological and semantic features.

\section{构式形态视角下英语 $x-f u 1$ 派生词形态语义互动研究}

\author{
陈晓静", 陈昌勇
}

\begin{abstract}
杭州电子科技大学，杭州，中国
“通讯作者. 邮箱：444843826@qq. com

中文摘要

在英语中后缀是重要的构词部件, “-ful” 作为常见的英语后缀之一, 产生的派生词数量众多。本文基于美国 当代英语语料库 (COCA), 提取 414例X-ful派生词, 对其形态、语义、并从构式形态学视角探究其形义互动 关系。研究发现: 1. x-ful派生词的词基可能为名词、形容词和动词, 能产生形容词和名词两类派生词; 2. x-ful 形容词派生词有十六种语义, 而 $\mathrm{x}-\mathrm{ful}$ 名词派生词只有一种; 3. X-ful形容词派生词的词基有四类语义范畴, 而 名词派生词则有八类。本研究有助于英语学习者了解X-ful派生词的语义图式, 正确把握其结构义, 理解其形 态和语义之间的关系，从而更好地掌握此类词汇。
\end{abstract}

关键词: X-ful派生词; 构式形态学; 语义特征; 形态特征; 形态与语义互动. 


\section{1. 引言}

英语后缀可以改变单词的词性或词义, 是一种重 要的构词部件。而-ful是常见的英语后缀, 产生的派 生词种类繁多、语义复杂。学者们对X-ful派生词进行 了相关的研究, 取得了富有意义的成果。许多学者首 先对X-ful派生词的语义进行了分析。（Stockwell\& Minkova, 2001; Andrew, 2002; Hamawand, 2007; Marchand, 1960；Plag，2003；）随着语言的发展， 越来越多的学者注意到它的复杂性, 他们从形态、词 缀对比、句法等不同的角度进行探索（Fabb，1987; Matthews，1991）。以上学者们基本上把形态和语义 分离成块进行研究, 对-ful派生词的形态和语义的互 动研究比较鲜见。后来, 认知语言学发展迅速, 很多 学者从认知的角度研究后缀, 但从此角度对-ful的研 究还未可见。鉴于此, 本文运用美国现代英语语料库

(COCA), 耖选出其中收录的x-ful派生词, 并以构 式形态学为理论框架, 研究其形态和语义特征以及形 义互动关系，以期揭示该类派生词的构词规则。

\section{X-ful派生词的语料库研究}

本文所选语料源于美国当代英语语料库（Corpus of Contemporary American English, 以下简称COCA）。 它是美国英语最大的平衡语料库, 收录了将近 5.6 亿英
语单词, 分为口语、小说、杂志、报纸、学术五类小 语料库。语料库最近一次更新是在 2019年，这为我们 的语料增加了时效性。

笔者搜集X-ful派生词的步骤如下: 首先, 在COCA 检索框输入“*ful”, 提取 $x$-ful语言数据。然后人工检 查各词, 排除后缀重复, 增添前缀的单词（如 supersuccessful, untrustful) 和一些拼写错误的词条 (如 ferdful）。接着, 对选取的X-ful派生词的词类, 语义, 以及词基的词类, 语义进行标注, 最后导入excel进行 分组。共计提取得出 414 个X-ful派生词。

\section{1. $X$-ful 派生词的形态特征}

为了全面考察后缀-ful对词基的选择，我们依据 COCA对-ful词基的词类分布进行了统计分析，得出 $\mathrm{x}-\mathrm{ful}$ 派生词的词基有名词、形容词和动词。名词派生 的词最多, 占例词总数的 $83.3 \%$ 。其次是动词, 所占 比例为 $12.8 \%$ 。而形容词词基只占 $3.9 \%$ 。

为了进一步探究 $\mathrm{x}-\mathrm{ful}$ 派生词的形态特征, 笔者详 细分析了 x-ful派生词词基的语义范畴。研究发现, $\mathrm{x}$-ful派生词词基共有 12 种语义范畴, 其中形容词派生 词的词基有四种语义范畴，名词性派生词词基则有八 种，具体情况见表1：

表1 X-ful派生词词基的语义范畴

\begin{tabular}{|c|c|c|c|c|}
\hline 派生词词类 & 词基语义范畴 & 数量 & 占比 & 例词 \\
\hline \multirow[b]{4}{*}{ 形容词 } & Abstract matters & 84 & $20.2 \%$ & $\begin{array}{l}\text { mindful ; } \\
\text { doomful }\end{array}$ \\
\hline & Emotion psychology & 79 & $19.0 \%$ & $\begin{array}{l}\text { plainful; } \\
\text { moanful }\end{array}$ \\
\hline & Action event & 26 & $6.5 \%$ & $\begin{array}{l}\text { provokeful; } \\
\text { wasteful }\end{array}$ \\
\hline & Property & 15 & 3.61\% & $\begin{array}{l}\text { beautiful; } \\
\text { lawful }\end{array}$ \\
\hline \multirow{8}{*}{ 名词 } & Container & 46 & $11.1 \%$ & pocketful ; tankful ; \\
\hline & Infrastructure & 41 & $9.9 \%$ & warehouseful; officeful; \\
\hline & Body & 33 & $7.9 \%$ & handful; lungful; \\
\hline & Tool & 27 & $6.5 \%$ & shovelful; spadeful; \\
\hline & Furniture & 19 & $4.6 \%$ & closetful; drawerful; \\
\hline & Transportation & 16 & $3.8 \%$ & truckful; carful; \\
\hline & Nature & 11 & $2.6 \%$ & treeful; riverful; \\
\hline & Animal & 2 & $0.4 \%$ & cowful; tentacleful ; \\
\hline
\end{tabular}

根据表1, -ful形容词词基的语义主要为抽象事物、 情绪及心理感受、动作事项和属性, 属于抽象性语义。 然而, $\mathrm{x}$-ful名词词基的语义主要为容器、基础设施、 人体、工具、家具、交通工具、自然和动物, 都具有 具体的形象。可见, 统计结果验证了 Plag (2003) 的 发现: 派生名词语义与抽象名词、动作结果、概念和
属性相联系，而派生形容词语义与具体的心理活动相 联系。

通过对不同词类的词基进一步的分析，我们发现， 动词词基的语义范畴有: 情感心理、动作事件, 其派 生词主要是形容词。动作可以表达主体的情绪, 即使 
语义范畴是单纯的动作事件，也能表达出一定的情感 倾向。名词词基的语义范畴最多, 主要有: 情感心理、 抽象事物、动作事件、属性、容器、家具、交通工具、 建筑、工具、动物、人体和自然等, 由此派生出形容 词和名词。大多数名词词基是属于容器范畴, 具有盛 装物体的功能。形容词词基的语义范畴有: 情感心理、 抽象事物和属性。以形容词为词基的派生词基本上是 形容词。以形容词为词基的 $x$-ful派生形容词表达的是 一种无形且不可触摸到的状态或感觉，与词基属于容 器范畴的x-ful派生名词属性相对。这类词汇主要表达 主体的情绪或状态。

\subsection{X-ful 的语义特征}

如何辨别 $\mathrm{x}$-ful派生词是名词还是形容词? 针对这 一问题, 学者们尚未进行深入地探讨。我们发现, 两 类派生词的语义特征有着明显的差别, 具体统计结果 见下表:

表2 X-ful派生词的语义特征

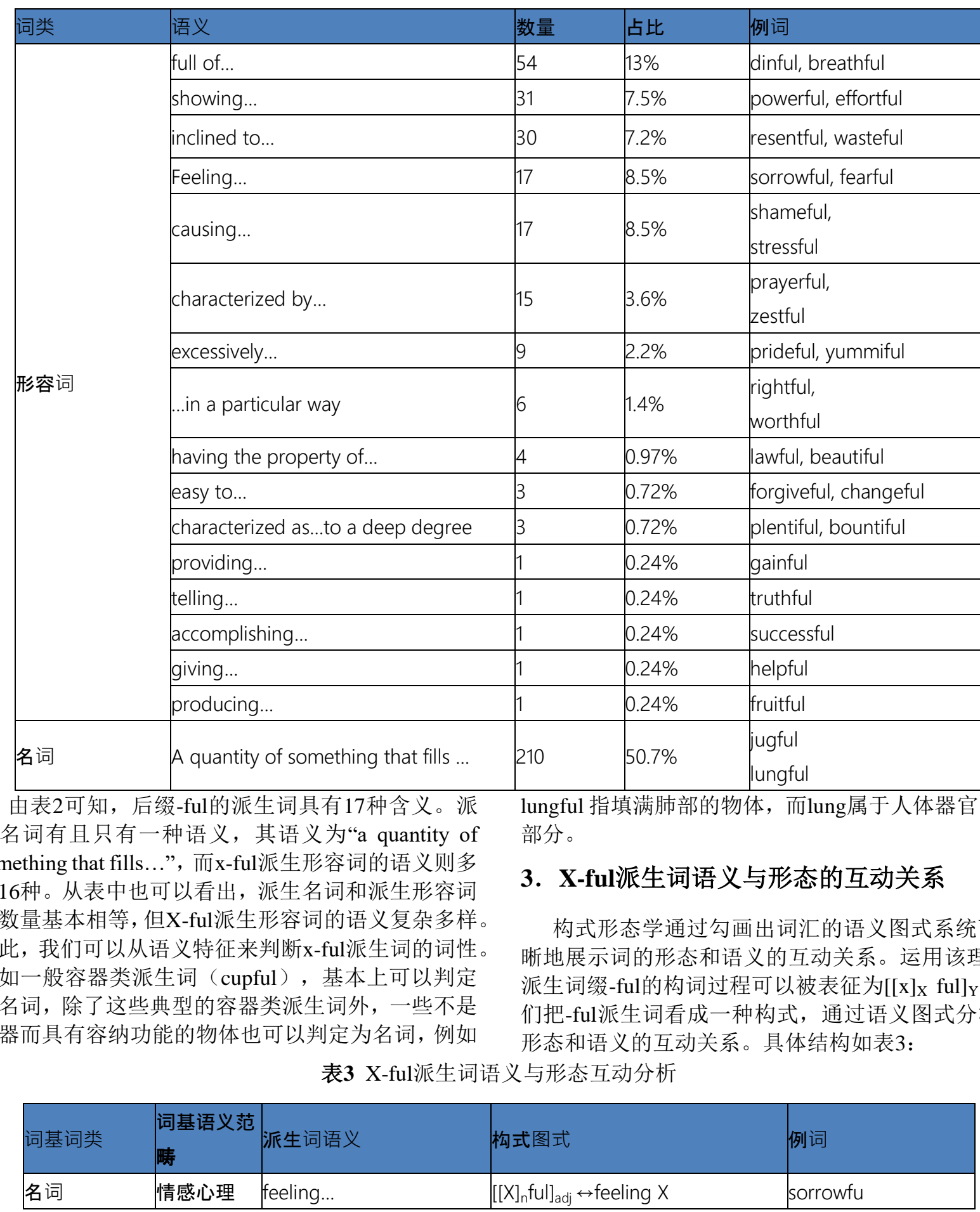




\begin{tabular}{|c|c|c|c|c|}
\hline & & causing... & {$\left[[\mathrm{X}]_{\mathrm{n}} \text { full }\right]_{\mathrm{adj}} \leftrightarrow$ causing $\mathrm{X}$} & delightful, \\
\hline & & full of... & {$\left[[X]_{n} \text { ful }\right]_{\text {adj }} \leftrightarrow$ full of $X$} & plainful, \\
\hline & & showing... & {$\left[[X]_{n} \text { ful }\right]_{\text {adj }} \leftrightarrow$ showing $X$} & merciful \\
\hline & & having... & {$\left[[X]_{n} \text { ful }\right]_{\text {adj }} \leftrightarrow$ having $X$} & graceful \\
\hline & & causing... & {$\left[[X]_{n} \text { full }\right]_{\text {adj }} \leftrightarrow$ causing $X$} & harmful \\
\hline & & full of... & {$\left[[X]_{n} \text { ful }\right]_{\text {adj }} \leftrightarrow$ full of X } & dinful \\
\hline & 抽象事物 & inclined to... & {$\left[[X]_{n} \text { ful }\right]_{\text {adj }} \leftrightarrow$ inclined to $X$} & slothful \\
\hline & & characterized by... & {$\left[[X]_{n} f u l\right]_{a d j} \leftrightarrow$ characterized by $X$} & prayerful \\
\hline & & showing... & {$\left[[X]_{n} f u l\right]_{a d j} \leftrightarrow$ showing $X$} & impactful \\
\hline & & providing... & {$\left[[X]_{n} f u l\right]_{a d j} \leftrightarrow$ providing $X$} & gainful \\
\hline & & telling... & {$\left[[X]_{n} \text { ful }\right]_{\text {adj }} \leftrightarrow$ telling $X$} & truthful \\
\hline & & inclined to... & {$\left[[X]_{n} \text { ful }\right]_{\text {adj }} \leftrightarrow$ inclined to $X$} & tearful \\
\hline & & accomplishing... & {$\left[[X]_{n} \text { ful }\right]_{a d j} \leftrightarrow$ accomplishing $X$} & successful \\
\hline & & producing... & {$\left[[X]_{n} \text { ful }\right]_{\text {adj }} \leftrightarrow$ producing $X$} & fruitful \\
\hline & & giving... & {$\left[[X]_{n} \text { ful }\right]_{\text {adj }} \leftrightarrow$ giving $X$} & helpful \\
\hline & & full of... & {$\left[[X]_{n} \text { ful }\right]_{\text {adj }} \leftrightarrow$ full of $X$} & rainful \\
\hline & 属性 & having the property of... & {$\left[[X]_{n} f u l\right]_{a d j} \leftrightarrow$ having the property of } & lawful \\
\hline & 容器 & & & bagsful \\
\hline & 家具 & & & deskful \\
\hline & 交通工具 & & & jeepful \\
\hline & 建筑 & a quantity of something & {$\left[[X]_{n} f u l\right]_{n} \quad \leftrightarrow \quad$ a quantity } & fwarehouseful \\
\hline & 工具 & that fills... & something that fills $X$ & shovelful \\
\hline & 动物 & & & cowful \\
\hline & 人体 & & & throatful \\
\hline & 自然 & & & riverful \\
\hline & & inclined to... & {$\left[[X]_{V} f u l\right]_{a d j} \leftrightarrow$ inclined to $X$} & resentful \\
\hline & 情感心理 & causing... & {$\left[[X]_{v} \text { ful }\right]_{\mathrm{adj}} \leftrightarrow$ causing $X_{n}$} & hurtful \\
\hline 动词 & & feeling ... & {$\left[[X]_{v} \text { ful }\right]_{\text {adj }} \leftrightarrow$ feeling $X_{\text {ing }}$} & scornful \\
\hline & & easy to... & {$\left[[X]_{v} f u l\right]_{\text {adj }} \leftrightarrow$ easy to X } & forgiveful \\
\hline & 动作事件 & causing... & {$\left[[X]_{\mathrm{V}} \mathrm{ful}\right]_{\mathrm{adj}} \leftrightarrow$ causing $\mathrm{X}_{\mathrm{n}}$} & wreckful \\
\hline & & inclined to... & {$\left[[X]_{\mathrm{V}} f u l\right]_{\mathrm{adj}} \leftrightarrow$ inclined to $\mathrm{X}$} & healful \\
\hline & 情感心理 & excessively... & {$\left[[\mathrm{X}]_{\mathrm{adj}} \mathrm{ful}\right]_{\mathrm{adj}} \leftrightarrow$ excessively $\mathrm{X}$} & proudful \\
\hline & & excessively... & {$\left[[X]_{\text {adj }} f u l\right]_{\text {adj }} \leftrightarrow$ excessively $X$} & yummiful \\
\hline 形空词 & 抽象事物 & ...in a particular way & {$\left[[X]_{\text {adj }} f u l\right]_{\text {adj }} \leftrightarrow X$ in a particular way } & worthful \\
\hline & 属性 & $\begin{array}{l}\text { characterized as... to } \\
\text { deep degree }\end{array}$ & {$\left[\begin{array}{l}\left.[\mathrm{X}]_{\mathrm{adj}} \mathrm{ful}\right]_{\mathrm{adj}} \leftrightarrow \text { characterized as } \mathrm{X} \text { to } \\
\text { a deep degree }\end{array}\right.$} & plentiful \\
\hline
\end{tabular}


由表3可知, x-ful派生词形态和语义相互影响。大 多数x-ful派生词的词基是名词, 少数是形容词和动词。 词基为名词时有 12 种语义范畴; 词基是动词时有 2 种 语义范畴；词基是形容词时有3种语义范畴。

名词主要指向容器功能。在 $x$-ful派生词中, 多数 名词派生词中有三种与词基密切相关的语义, 分别是 情绪感觉, 抽象事物和容器功能, 其中容器功能更加 凸显。这其中，有且只有这一类名词词基所构成的派 生词为 $\mathrm{x}$-ful派生名词, 即可以存储东西的容器。例如, jarful是指一定数量的糖果装满了整个罐子。对 $\mathrm{x}$-ful 派生词来说, 要想产生容器功能的语义, 最好的选择 就是具有一定空间能够容纳物体的名词, 这样构成的 派生词无论是词性或者语义在认知上会具有更高的 辨识度。

动词表达心理情绪和动作。词基为动词的派生词 共有53个, 我们发现其中 30 个派生词语义属于情感心 理范畴。当词基为动词时, 派生词语义由动词词基语 义范畴决定。判断来源于动词词基带有丰富的心理情 绪和动作, 例如文中提到的 forgiveful, 词基为forgive, 派生词语义也隐含着与词基forgive一样的动作指向。

形容词主要修饰人和物的特征。在词基为形容词 的 $\mathrm{x}$-ful形容词中, 词基形容词和派生词有以下三种关 系: 1. 当主体是人时, 词基语义能够表达其情绪, 与 动词词基的派生词语义相似, 只是情绪程度不同。例 如语料中的派生词proudful, proud为形容词, 派生词 语义为极度自豪的, 这其实是主体人的自豪感。2. 当 主体是实物时, 派生词语义是形容一个物体的属性, 例如sourful, 词基是形容词sour, 派生词语义是酸的, 其描述的是某物酸的属性。3. 当人对事物进行主观的 评价时, 派生词语义是主体对物体的一个超常感受, 例如yummiful, 词基是形容词yummy, 派生词语义是 极度美味的, 其语义扩大了主体对事物的一个感受。

\section{4. 结语}

本文基于美国当代英语语料库（COCA），探讨了 $\mathrm{x}$-ful派生词的形态及语义特点, 并从构式形态学视角 探究其形态与语义互动关系。研究发现, X-ful派生词 词类简单, 主要和名词、形容词、动词搭配形成x-ful 派生名词和形容词, 但形态复杂, 词基涉及 12 种语义 范畴。至于语义方面, $\mathrm{x}$-ful的语义也十分丰富, 共有 17种语义。最后就形态语义关系而言, x-ful派生词构 式图式多样, 不同的语义和形态互动明显, 形义相互 影响, 相互制约。并且x-ful派生词图式能使英语学习 者更好的了解 $\mathrm{x}$-ful派生词形成规律和特征, 从而促进 此类英语词汇的学习和教学。

\section{致谢}

在此论文完成之际, 我要感谢我的老师陈昌勇副 教授, 多次见面讨论写作, 给予了我极大地鼓励和指 导，使得我的论文能够顺利完成。同时感谢我的家人
和朋友们, 让我能专心完成此篇文稿。再次感谢大家 对我的帮助和支持!

最后，诚挚地感谢各位评阅本论文的专家教授， 感谢你们的耐心评阅！

\section{REFERENCES}

[1] Booij,G. Construction Morphology[J]. John Wiley \& Sons, Ltd (10.1111),2010.

[2] Booij,G. Construction Morphology, a brief introduction[J]. Springer Netherlands,2012.

[3] Mccarthy,A.C. An Introduction to English Morphology: Words and Their Structure[M]. Edinburgh: EUP, 2002

[4] Hamawand,Z. Suffixal Rivalry in Adjective Formation-A cognitive-corpus analysis[M]. London: Equinox Publishing Ltd., 2007.

[5] Marchand,H. The Categories and Types of Present-Day English Word-Formation: A Synchronic-Diachronic Approach[M]. Munich: Verlag C. H. Beck,1969.

[6] Plag, I. Word-Formation in English[M]. Cambridge: Cambridge University Press, 2003.

[7] Fabb,N. English suffixation is constrained only by selectional restrictions[J]. Kluwer Academic Publishers, 1988.

[8] Matthews,P.H. Morphology[M]. New York, Cambridge University Press, 1991.

[9] Stockwell,R.\& Minkova,D. English Words: History and Structure[M]. New York, Cambridge University Press, 2001.

[10] Fitryannisa P. A Morphological Analysis of the Suffix $\{$-ful $\}$ in Forming New Lexical Categories[D]. Yogyakarta, Sanata Dharma University, 2017.

[11] Yishen Shao.The Root Difference between English ful nominal and adjective Derivatives [J].Masterpieces Review,2016(32):65-66+113. 